\section{PTH-050 DO ANTITHROMBOTIC MEDICATIONS AFFECT THE OUTCOMES OF ACUTE UPPER GASTROINTESTINAL BLEEDING?}

Yusuke Onishi*, Wilson Siu, Balasubramaniam Vijayan. Department of Gastroenterology, Aberdeen Royal Infirmary, NHS Grampian, Aberdeen, UK

\subsection{6/gutjnl-2019-BSGAbstracts.75}

Background There is a widespread use of antithrombotic (AT) medications in primary and secondary prevention of cardiovascular disease in recent years, particularly since the introduction of direct oral anticoagulants (DOACs).

The aim of our study is to determine clinical predictors of adverse outcome in patients with non-variceal upper GI bleeding comparing the patients who were on AT drugs to those who were not any.

Methods All patients admitted to the bleeding unit with a primary diagnosis of upper GI bleeding between 01/05/2015 to $15 / 09 / 2017$ were identified. Variceal bleedings were excluded in this study. Patients' demographics, AT prescriptions, co-morbidities, admission time and blood results on the day of admission were collected. Outcomes of the study included 30day mortality, endoscopic therapy, blood transfusion, re-bleeding (from the same admission) and duration of hospital stay. The relationship between the variables and clinical outcomes were examined.

Results 291 patients (Median age: 71, IQR: 59-81, male: $66.7 \%)$ were analysed and peptic ulcer bleeding was the commonest cause of bleeding (56.7\%). 94\% of endoscopies were performed within 24 hours of admission. Endoscopic treatment and blood transfusion were required in $49 \%$ and $59 \%$ respectively, whilst $13 \%$ of cases required further radiological/ surgical interventions. Re-bleeding rate was $7.5 \%$, medial length of stay was 6 days (IQR 4-11), and all-cause mortality rate was $8.9 \%$.

The number of patients on NSAIDs/Aspirin, Clopidogrel/ Ticagrelor, Warfarin, and DOACs were 99, 26, 33, and 16 respectively. There was no statistically significant difference in all clinical outcomes in patients on ATs versus those not on any. After logistic regression analysis, raised C-reactive Protein (CRP, $\mathrm{p}=0.003)$ and co-morbidities $(\mathrm{p}=0.038)$ were significant predictors of 30-day mortality. Male gender $(p=0.021)$ was a significant predictor of need for endoscopic treatment. Bleeding with a fall in haemoglobin $(\mathrm{Hb}$, $\mathrm{p}<0.005$ ) was a significant predictor for need for blood transfusion. Finally, endoscopy undertaken within 24 hours of admission $(\mathrm{p}<0.005)$ and normal $\mathrm{Hb}(\mathrm{p}=0.015)$ shortened the duration of admission with statistical significance, while prolonged prothrombin time $(\mathrm{p}=0.026)$ and raised CRP $(p=0.034)$ correlated significantly with prolonged duration of hospital stay.

Conclusion We found no significant difference in all clinical outcomes in patients on different ATs compared to patients who are not. Out-of-hours admissions did not have any significant influence on clinical outcomes either.

\section{PTH-051 EYE GAZE-CONTROLLED ROBOTIC FLEXIBLE ENDOSCOPY: A FEASIBILITY STUDY}

Nisha Patel*, Alexandros Kogkas, Ara Darzi Ben Glover, George Mylonas. Imperial College London, London, UK

\subsection{6/gutjnl-2019-BSGAbstracts.76}

Introduction A gaze-controlled robotic endoscope is innovative technology with myriad potential applications in the rapidly advancing field of flexible endoscopy. Improvements to the current flexible device to allow examination of the gastrointestinal tract whilst minimising procedural discomfort and complications are desirable.

Methods Users are able to control endoscope movements without handling the device. A flexible gastroscope (Karl Storz 13801 PKS) was attached to a UR5 6 axis robotic arm (Universal Robots), mounted onto a rail and placed on top of a surgical table. Two cogwheel shaped dials were 3D printed and placed onto the up/down and left/right wheels on the head of the gastroscope (Figure 1a). Robotization of these controls was achieved by using two motors (DYNAMIXEL RX-24F) to steer the distal tip.

This system allows users to operate a robotised flexible endoscope using eye gaze control. Gaze interaction with the screen was based on a 3D gaze framework we developed with the synergy of conventional wearable eye-tracking glasses, a motion capture system and fixed in space RGB-D cameras for $3 \mathrm{D}$ reconstruction of the environment. The eye gaze technology was calibrated for each user prior to the task.

The distal tip of the gastroscope was controlled using eye gaze technology. The UR5 robot was used to enable shaft rotation. This was initiated by fixed head movements left and right rotating the shaft clockwise/anticlockwise. Linear movements of the gastroscope were triggered using a joystick handle (up for forward movement, down for endoscope withdrawal). Pause and automatic retroflexion of the endoscope are achieved by moving the joystick left and right respectively.

Users were asked to navigate an endoscope through an UGIT model (Chamberlain group) simulating a diagnostic

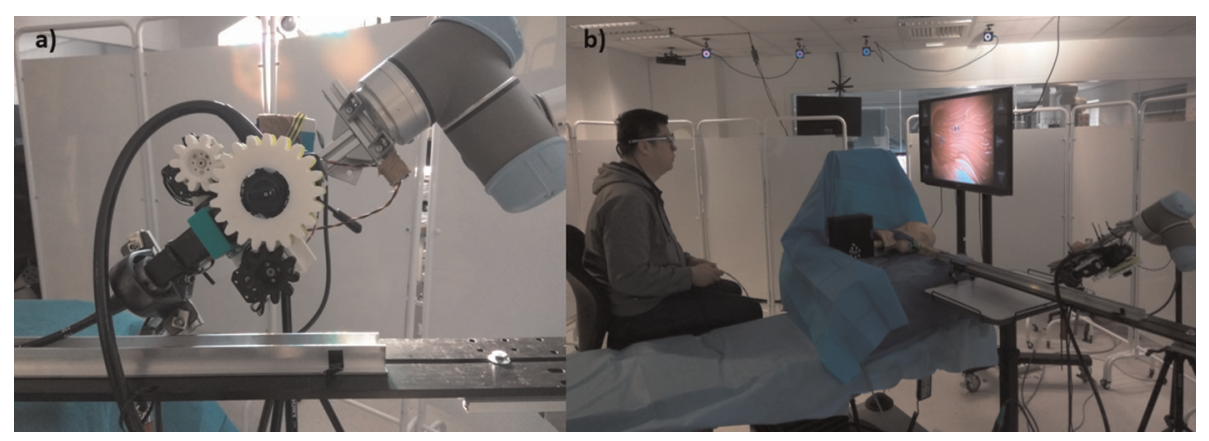

Abstract PTH-051 Figure 1 (a) 3D printed dials (b) study set-up 